\title{
Programa de boas práticas em domicílios da cidade de Santa Maria - RS
}

\author{
Program good practice in households of the city of Santa Maria - RS
}

\section{Autores | Authors}

凶 Barbara Cecconi DEON

Instituto Federal Farroupilha

Curso Técnico em Alimentos

Rua 20 de Setembro, s/n

CEP: $97420-000$

São Vicente do Sul/RS - Brasil

e-mail: bcdeon@hotmail.com

\section{Luiza Helena HECKTHEUER}

Universidade Federal de Santa Maria (UFSM)

Departamento de Ciência e Tecnologia dos Alimentos

Santa Maria/RS - Brasil

e-mail: luihrh@hotmail.com

Mariana Araújo ETCHEPARE

Universidade Federal de Santa Maria (UFSM)

Departamento de Ciência e Tecnologia dos Alimentos

Santa Maria/RS - Brasil

e-mail: marianaaetchepare@hotmail.com

Maritiele NAISSINGER

Universidade Federal de Santa Maria (UFSM)

Departamento de Ciência e Tecnologia dos Alimentos

Curso de Nutrição

Santa Maria/RS - Brasil

e-mail:maritielens@gmail.com

Silvana SACCOL

Universidade Federal de Santa Maria (UFSM)

Departamento de Ciência e Tecnologia dos Alimentos

Santa Maria/RS - Brasil e-mail: silvanasaccol@gmail.com

Autor Correspondente / Corresponding Author

Publicado / Published: dezembro/2012

\section{Resumo}

Os alimentos preparados nos domicílios têm grande influência na ocorrência de surtos de Doenças Transmitidas por Alimentos, como resultado de falhas higiênicas na manipulação dos alimentos. Dessa forma, este trabalho teve como objetivo desenvolver e aplicar um programa educativo sobre boas práticas na manipulação de alimentos nos domićlios. O estudo foi realizado em 615 domicílios da cidade de Santa Maria-RS, no ano de 2011, e foi dividido em três etapas: diagnóstico; elaboração e aplicação do programa educativo; avaliação do acesso ao programa. Verificou-se que $49 \%$ da amostra pesquisada teve acesso ao programa educativo e a região nordeste do município de Santa Maria-RS foi a que acompanhou o programa com mais frequência. A partir dos resultados, sugere-se a necessidade de continuidade de programas no processo de conscientização da população, visto que os domicílios são um elo fundamental na cadeia alimentar para prevenir as doenças de origem alimentar, buscando-se, assim, a melhoria da qualidade de vida da coletividade.

Palavras-chave: Doenças transmitidas por alimentos; Boas práticas de manipulação; Diagnóstico; Lista de avaliação; Educação.

\section{Summary}

The food prepared at home has had a great influence on the occurrence of Foodborne Disease Outbreaks as a result of hygienic failures in the food manipulation. Thus, this work aimed to develop and apply an education program on good practice for the manipulation of foods at home. This study was conducted in 615 houses in the city of Santa Maria-RS in 2011 and divided into three stages: diagnosis; development and implementation of the educational program, and the evaluation of the access to the program. It was found that $49 \%$ of the sample surveyed had access to the educational program which was more frequently followed by the northeast region. These results suggest the need to continue with programs on the consciousness-raising process of the population since homes are a fundamental link in the food chain to prevent foodborne diseases seeking an improvement of the quality of life of the population.

Key words: Foodborne diseases; Good practice for manipulation; Diagnosis; Evaluation list; Education. 


\section{Introdução}

A alimentação, para Proença et al. (2005), é uma das atividades mais importantes do ser humano, tanto por razões biológicas quanto pelas questões sociais e culturais que envolvem o ato de comer. Assim, alimentar-se engloba vários aspectos, que vão desde a produção dos alimentos até a sua transformação em refeições e disponibilização às pessoas.

As transformações no mundo contemporâneo provocaram mudanças significativas na alimentação, no preparo das refeições e nos hábitos alimentares dos seres humanos. Essas mudanças foram ocasionadas por fatores que perpassam a urbanização, a industrialização, a profissionalização das mulheres, a elevação do nível de vida e de educação, o acesso mais amplo da população ao lazer, a redução do tempo para o preparo e/ou consumo do alimento, entre outros fatores, os quais estão envolvidos diretamente na manipulação e na preparação dos alimentos (KARABUDAK et al., 2008).

A cada ano, pelo menos dois bilhões de pessoas no mundo sofrem de doenças de origem alimentar, tornando-se este um dos maiores problemas de saúde pública no mundo contemporâneo (BEHRENS et al., 2010). No Brasil, segundo dados da Análise Epidemiológica dos Surtos de Doenças Transmitidas por Alimentos, no período de 2000 a 2011, 51,8\% (3746 casos) dos locais de ocorrência dos surtos de Doenças Transmitidas por Alimentos (DTAs) tiveram origem domiciliar, estando acima de todos os outros locais, inclusive restaurantes. Dos surtos notificados, as regiões Sul, Sudeste e CentroOeste foram as mais expressivas no país (BRASIL, 2011).

Redmond e Griffith (2003) apontam que muitos casos de DTAs poderiam ser evitados se comportamentos preventivos fossem adotados em toda a cadeia produtiva de alimentos. No entanto, diante da impossibilidade de produzir alimentos totalmente isentos de patógenos, os cuidados na manipulação dos alimentos, nos domicílios, representam, hoje, importante etapa para reduzir a incidência dessas doenças (UNUSAN, 2007). Estudos mostram que grande parte das pessoas que preparam alimentos em casa está mal informada sobre as medidas necessárias para prevenir estas doenças (KARABUDAK et al., 2008).

Unusan (2007) complementa que não existe uma regulamentação para a preparação, a manipulação e o armazenamento de alimentos em casa e, em virtude da crescente preocupação com o aumento dos casos de DTAs nos domicílios, há necessidade de programas serem desenvolvidos, sensibilizando os consumidores para adoção de boas práticas na manipulação de alimentos.

Tendo em vista o exposto, fazem-se necessários mais estudos com esta população, visto que os domicílios são um elo fundamental na cadeia alimentar para prevenir as DTAs. A partir dessas expectativas, objetivou-se desenvolver e aplicar um programa educativo sobre boas práticas na manipulação de alimentos nos domicílios.

\section{Material e métodos}

O presente estudo foi dividido em três etapas, com uma amostra de 615 domicílios distribuídos nos 41 bairros da cidade de Santa Maria-RS, os quais estão agrupados em Regiões Administrativas (RA). Pela Lei Complementar n. ${ }^{\circ}$ 042, de 29 de dezembro de 2006, a divisão geográfica da zona urbana de Santa Maria está representada por oito Regiões Administrativas: Centro Urbano; Norte; Nordeste; Leste; Centro-Leste; Sul; Centro-Oeste, e Oeste (SANTA MARIA, 2006). Em razão de a Região Leste apresentar somente um bairro e para melhorar a acurácia dos dados, este foi agrupado juntamente com a região Centro-Leste, perfazendo, assim, sete RAs. Como critérios de inclusão da amostra, foram utilizados: i) interesse e disponibilidade em participar da pesquisa; ii) presença do responsável pela manipulação dos alimentos no momento da entrevista, e iii) permissão para avaliar a cozinha.

Na primeira etapa, o diagnóstico, no período de março a julho de 2011, aplicou-se um formulário ao responsável pela manipulação de alimentos nos domicílios, abrangendo dados pessoais e específicos de boas práticas na manipulação dos alimentos. Para enriquecer a pesquisa, foi realizada uma avaliação visual nas cozinhas domiciliares por meio da aplicação de uma Lista de Avaliação das Boas Práticas nos Domicílios.

Nos meses de agosto a outubro de 2011, a segunda etapa, com base nas informações coletadas e nas principais dificuldades dos manipuladores nos domicílios, foi realizado o programa educativo, através dos meios de comunicação de maior acesso relatados durante a aplicação do formulário, como: rádio, palestras nos bairros, televisão, jornal e pela internet (facebook e e-mails). Como material facilitador de aprendizagem, foi elaborada a Cartilha do Manipulador de Alimentos nos Domicílios, a qual foi construída levando-se em consideração as práticas de manipulação de alimentos seguros.

Na terceira e última etapa da pesquisa, no mês de novembro de 2011, após aplicação do programa educativo, foi avaliado o acesso desse programa, retornado a um terço dos entrevistados de cada bairro, totalizando 205 domicílios.

Para determinar o acesso dos entrevistados ao programa educativo, utilizaram-se estatísticas descritivas de frequência. O intervalo de confiança estimado foi de 95\% e a análise estatística desenvolvida foi realizada por meio do software Statistical Package for Social Science 15.0 (SPSS). 
Programa de boas práticas em domicílios da cidade de Santa Maria - RS

DEON, B. C. et al.

\section{Resultados e discussão}

Na Figura 1, pode-se verificar o acesso dos participantes ao programa educativo. Da amostra pesquisada ( $n=205), 49 \%(n=84)$ viabilizaram 0 programa, enquanto $51 \%(n=121)$ não tiveram acesso. Embora o percentual de acessibilidade ao programa tenha sido baixo, quando comparado com os que não tiveram acesso ainda foi um número expressivo, ao se analisar o pouco tempo do programa, que ocorreu de agosto a outubro de 2011. Soto et al. (2009), em pesquisa semelhante, na etapa do programa educativo, veicularam mensagens informativas nas sacolas do supermercado parceiro com orientações referentes à segurança dos alimentos nos domicílios, durante dez meses.

Segundo a Figura 2, da amostra que teve acesso ao programa educativo, 33\% $(n=28)$ tiveram acesso somente uma vez ao programa, 39\% ( $n=33)$ tiveram acesso entre 2 e 5 vezes, e $28 \%(n=23)$ acompanharam o programa mais de 5 vezes.

Constatou-se que os entrevistados da Região Nordeste foram os que acompanharam com mais frequência o programa educativo, por mais de 5 vezes (46\%). A RA Centro-Leste apareceu com frequência maior, de 2 a 5 vezes (60\%), e a região que menos acompanhou o programa foi a Sul, pois a maioria (50\%) dos indivíduos teve acesso somente uma vez.

Conforme mostra a Tabela 1, dos 84 entrevistados que tiveram acesso ao programa educativo, 100\% informaram ter gostado de receber as informações. Quanto à questão "Colocou em prática as informações recebidas?", 88\% relataram ter colocado em prática muitas informações recebidas ou que estão procurando aplicar, aos poucos, os conhecimentos adquiridos, ressaltando ainda que tudo foi válido; $12 \%$ indicaram

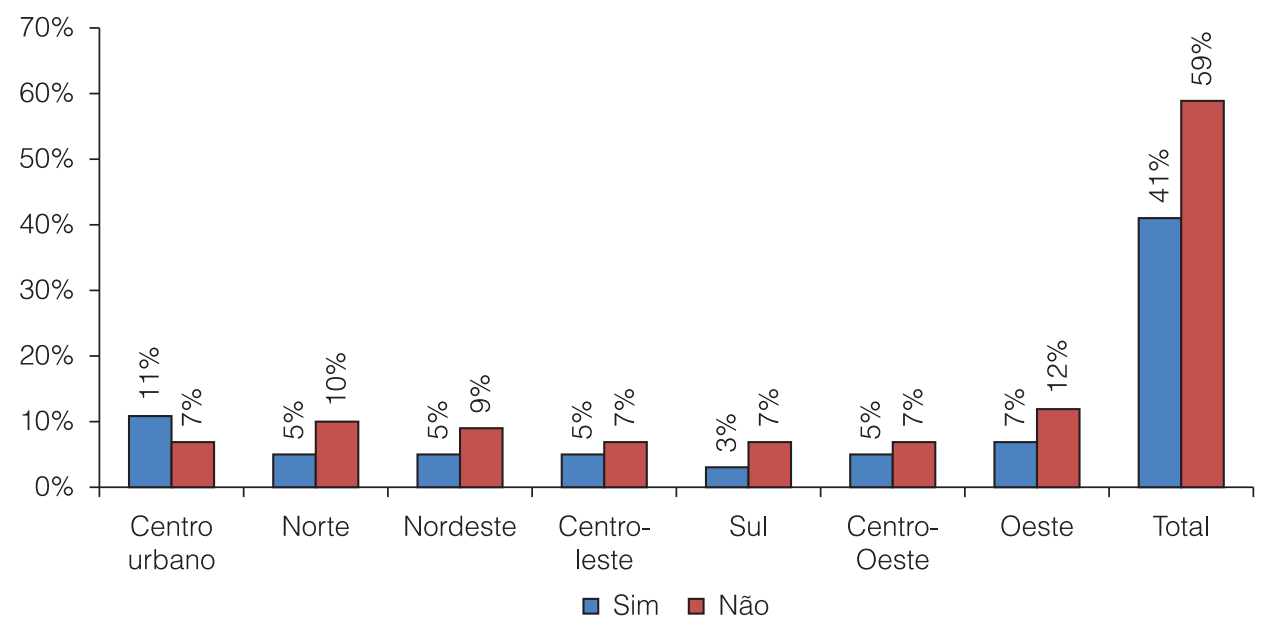

Figura 1. Avaliação da questão "acesso a algum programa educativo" pelos manipuladores de alimentos nos domicílios da cidade de Santa Maria-RS (Freq. \%), 2011.

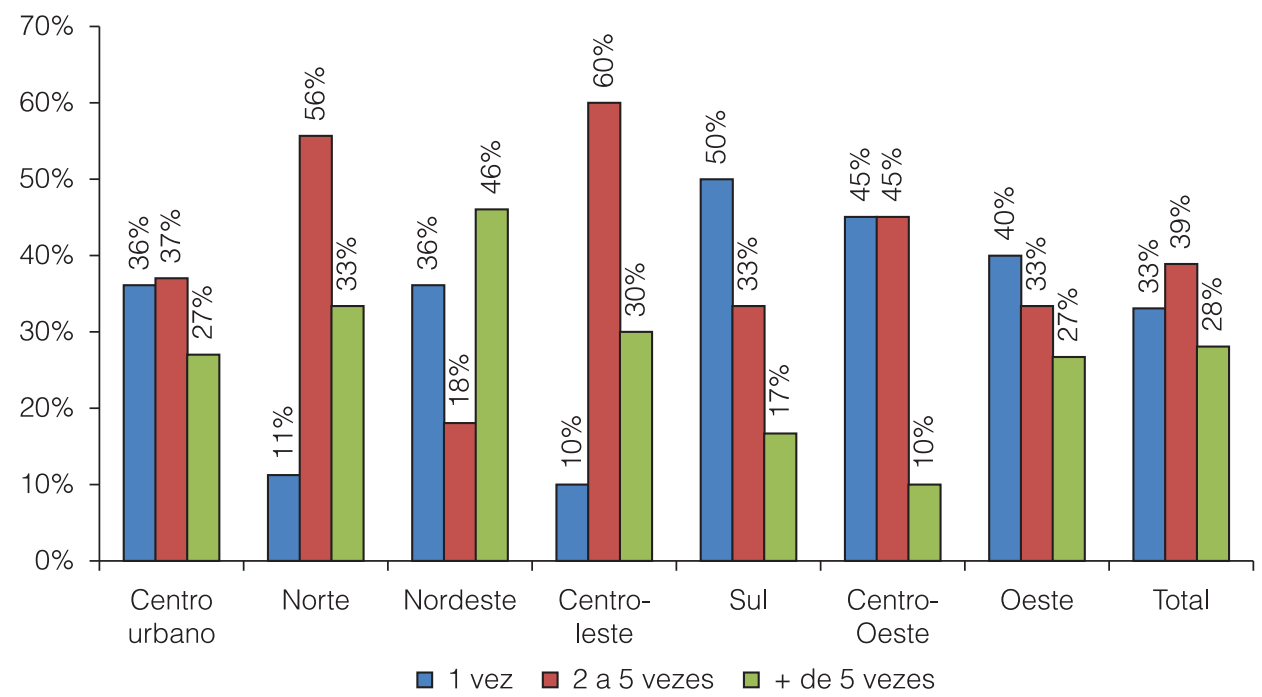

Figura 2. Avaliação da questão "Quantas vezes teve acesso ao programa educativo?" pelos manipuladores de alimentos nos domicílios da cidade de Santa Maria-RS (Freq. \%), 2011. 
Programa de boas práticas em domicílios da cidade de Santa Maria - RS

DEON, B. C. et al.

Tabela 1. Avaliação das questões "Gostou de receber as informações?" e "Colocou em prática as informações recebidas?" pelos manipuladores de alimentos nos domicílios da cidade de Santa Maria-RS (Freq. \%), 2011.

\begin{tabular}{lcc}
\multicolumn{1}{c}{ Questões } & Sim (\%) & Não (\%) \\
Gostou de receber as informações? & 84 & 0 \\
& $(100,0)$ & $(0,0)$ \\
Colocou em prática as informações & 74 & 10 \\
recebidas? & $(88,0)$ & $(12,0)$ \\
\hline
\end{tabular}

não ter colocado as informações em prática por motivos diversos, como, por exemplo, falta de tempo, já tinham conhecimento das informações anteriormente, entre outros.

Esse resultado, mesmo que isolado, já é importante, porque contribuiu para que alguma mudança de conduta em relação à higiene de alimentos nos domicílios fosse implementada, a fim de colaborar com a segurança dos alimentos consumidos pela população. Há de ser destacada, nesta pesquisa, a utilização dos meios de comunicação, uma vez que as informações foram transmitidas para um grande público, não necessariamente somente para a amostra pesquisada.

Candeias (1997), em sua pesquisa, justificou que a mudança no comportamento individual após uma ação educativa beneficia, de um modo geral, toda a população com a qual este indivíduo encontra-se envolvido. Conforme Scott (1996) concluiu em seu estudo, os benefícios da educação em higiene e o desenvolvimento de uma política de higiene domiciliar podem não só reduzir a ocorrência de DTAs, mas também conscientizar as pessoas acerca dos princípios de higiene na comunidade em geral, como creches, escolas e restaurantes.

A implementação de programas educativos sobre a manipulação dos alimentos é fundamental, visto que a qualidade higiênico-sanitária dos mesmos constitui um fator essencial à segurança dos alimentos (TAVOIARO et al., 2006).

\section{Conclusão}

A partir dos resultados deste trabalho, observou-se que o programa educativo foi bem aceito e comentado por todos aqueles que a ele tiveram acesso. Tal universo não se constituiu necessariamente da amostra pesquisada, mas também pelos parceiros dos meios de comunicação, que reconheceram a relevância do programa pelos assuntos trabalhados e por ter atingido uma grande população. Sugere-se a necessidade de continuidade de programas no processo de conscientização da população, visto que os domicílios são um elo fundamental na cadeia alimentar para prevenir as DTAs, buscando-se, dessa forma, a melhoria da qualidade de vida da coletividade. Ressalta-se, também, a utilização dos meios de comunicação como ótimos parceiros, podendo este modelo de trabalho ser aplicado para outros tipos de campanhas informativas que envolvam a segurança dos alimentos nos municípios.

\section{Referências}

BEHRENS, J. H.; BARCELLOS, M. N.; FREWER, L. J.; NUNES, T. P.; FRANCO, B. D. G. M.; DESTRO, M. T.; LANDGRAF, M. Consumer purchase habits and views on food safety: A Brazilian study. Food Control, Guildford, v. 21, n. 7, p. 963-969, July 2010. http://dx.doi.org/10.1016/j.foodcont.2009.07.018

BRASIL. Ministério da Saúde. Dados epidemiológicos - DTA período de 2000 a 2011*. Brasília: SVS, 2011. Disponível em: <http://portal.saude.gov.br/portal/arquivos/pdf/10_passos_ para_investigacao_surtos.pdf>. Acesso em: 14 out. 2011.

CANDEIAS, N. M. F. Conceitos de educação e de promoção em saúde: mudanças individuais e mudanças organizacionais. Revista Saúde Pública, São Paulo, v. 31, n. 2, p. 209-213, abr. 1997

KARABUDAK, E.; BAS, M.; KIZILTAN, G. Food safety in the home consumption of meat in Turkey. Food Control, Amsterdam, v. 19, n. 3, p. 320-327, Mar. 2008. http://dx.doi.org/10.1016/j. foodcont.2007.04.018

PROENÇA, R. P. C.; SOUSA, A. A.; VEIROS, M. B.; HERING, B. Qualidade Nutricional e Sensorial na Produção de Refeições. Florianópolis: Ed. UFSC, 2005.

REDMOND, E. C.; GRIFFITH C. J. Consumer food handling in the house: a review of food safety studies. Journal of Food Protection, Des Moines, v. 66, n. 1, p. 130-161, Jan. 2003.

SANTA MARIA. Prefeitura Municipal. Lei complementar $n^{\circ} 042$, de 29 de dezembro de 2006. Cria unidades urbanas, altera a divisão urbana de Santa Maria, dá nova denominação aos bairros e revoga a Lei Municipal n²770/86, de 02/07/1986, Artigos $2^{\circ}$ a 25 e dá outras providências. Diário Oficial do Município de Santa Maria, Santa Maria, RS, 30 dez. 2006. Disponível em: <http://www.santamaria.rs.gov.br/docs/secretarias/ArqSec60. pdf>. Acesso em: 25 out. 2010.

SCOTT, E. Foodborne disease and other hygiene issues in the home. Journal of Applied Bacteriology, Oxford, v. 80, n. 1, p. 5-9, 1996. PMid:8698654. http://dx.doi. org/10.1111/j.1365-2672.1996.tb03181.x

SOTO, F. R. M.; OLIVEIRA, E.; HIDEKI, E.; LÚCIO, D.; CAMARGO, S. R.; BALIAN, S. C. Programa de vigilância sanitária de alimentos em domicílios no município de Ibiúna, SP. Higiene Alimentar, São Paulo, v. 23, n. 176-177, p. 64-67, set./out. 2009.

TAVOIARO, P.; OLIVEIRA, C. A. F.; LEFEVRE, F. Avaliação do conhecimento em práticas de higiene: uma abordagem qualitativa. Interface: Comunicacao, Saude, Educacao, Botucatu, v. 18, n. 9, p. 243-54, 2006. http://dx.doi.org/10.1590/ S1414-32832006000100017

UNUSAN, N. Consumer food safety knowledge and practices in the home in Turkey. Food Control, Guildford, v. 18, n. 1, p. 45-51, Jan. 2007. http://dx.doi.org/10.1016/j.foodcont.2005.08.006 